\title{
Data Shepherding in Nanotechnology : The Exposure Field Campaign Template
}

\author{
Furxhi, Irini
}

2021-07-13

Furxhi , I , Koivisto , A J , Murphy , F , Trabucco , S, Del Secco , B \& Arvanitis , A 2021 , ' Data Shepherding in Nanotechnology : The Exposure Field Campaign Template ' , Nanomaterials , vol. 11 , no. 7 , 1818 . https://doi.org/10.3390/nano11071818

http://hdl.handle.net/10138/333634

https://doi.org/10.3390/nano11071818

cc_by

publishedVersion

Downloaded from Helda, University of Helsinki institutional repository.

This is an electronic reprint of the original article.

This reprint may differ from the original in pagination and typographic detail.

Please cite the original version. 


\title{
Article \\ Data Shepherding in Nanotechnology. The Exposure Field Campaign Template
}

\author{
Irini Furxhi ${ }^{1,2, *(\mathbb{D})}$, Antti Joonas Koivisto ${ }^{3,4,5} \mathbb{D}$, Finbarr Murphy ${ }^{1,2} \mathbb{D}$, Sara Trabucco ${ }^{6}$, Benedetta Del Secco ${ }^{6} \mathbb{D}$ \\ and Athanasios Arvanitis ${ }^{7}$
}

check for updates

Citation: Furxhi, I.; Koivisto, A.J.; Murphy, F.; Trabucco, S.; Del Secco, B.; Arvanitis, A. Data Shepherding in Nanotechnology. The Exposure Field Campaign Template. Nanomaterials 2021, 11, 1818. https://doi.org/ $10.3390 /$ nano11071818

Academic Editor: Nicola Calabretta

Received: 15 June 2021

Accepted: 9 July 2021

Published: 13 July 2021

Publisher's Note: MDPI stays neutral with regard to jurisdictional claims in published maps and institutional affiliations.

\section{Copyright: (c) 2021 by the authors.} Licensee MDPI, Basel, Switzerland. This article is an open access article distributed under the terms and conditions of the Creative Commons Attribution (CC BY) license (https:/ / creativecommons.org/licenses/by/ $4.0 /)$.
1 Transgero Limited, Cullinagh, Newcastle West, V42V384 Limerick, Ireland; Finbarr.murphy@transgero.eu

2 Department of Accounting and Finance, Kemmy Business School, University of Limerick, V94T9PX Limerick, Ireland

3 Air Pollution Management, Willemoesgade 16, st tv, DK-2100 Copenhagen, Denmark; joonas.apm@gmail.com 4 ARCHE Consulting, Liefkensstraat 35D, B-9032 Wondelgem, Belgium

5 Institute for Atmospheric and Earth System Research (INAR), University of Helsinki, PL 64, FI-00014 Helsinki, Finland

6 Institute of Atmospheric Sciences and Climate (CNR-ISAC) Via Gobetti 101, 40129 Bologna, Italy; s.trabucco@isac.cnr.it (S.T.); b.delsecco@isac.cnr.it (B.D.S.)

7 Environmental Informatics Research Group, Department of Mechanical Engineering, Aristotle University of Thessaloniki, 54124 Thessaloniki, Greece; tharvanitis@meng.auth.gr

* Correspondence: irini.furxhi@transgero.eu

\begin{abstract}
In this paper, we demonstrate the realization process of a pragmatic approach on developing a template for capturing field monitoring data in nanomanufacturing processes. The template serves the fundamental principles which make data scientifically Findable, Accessible, Interoperable and Reusable (FAIR principles), as well as encouraging individuals to reuse it. In our case, the data shepherds' (the guider of data) template creation workflow consists of the following steps: (1) Identify relevant stakeholders, (2) Distribute questionnaires to capture a general description of the data to be generated, (3) Understand the needs and requirements of each stakeholder, (4) Interactive simple communication with the stakeholders for variables/descriptors selection, and (5) Design of the template and annotation of descriptors. We provide an annotated template for capturing exposure field campaign monitoring data, and increase their interoperability, while comparing it with existing templates. This paper enables the data creators of exposure field campaign data to store data in a FAIR way and helps the scientific community, such as data shepherds, by avoiding extensive steps for template creation and by utilizing the pragmatic structure and/or the template proposed herein, in the case of a nanotechnology project (Anticipating Safety Issues at the Design of Nano Product Development, ASINA).
\end{abstract}

Keywords: nanotechnology; exposure assessment; field campaigns; FAIR data; data management plan

\section{Introduction}

The incorporation of nano-objects (NOs) (https: / / www.iso.org/obp/ui/\#iso:std:iso: ts:80004:-1:ed-1:v1:en:term:2.5 (accessed on 5 June 2021)) to an increasing number of nanostructured materials (https://www.iso.org/obp/ui/\#iso:std:iso:ts:80004:-4:ed-1:v1:en (accessed on 5 June 2021)) worldwide is driven by the benefits of novel applications [1,2]. The possible health effects derived from the exposure to these new NOs are still ambiguous [3]. Hazard identification and exposure assessments (occupational and environmental) are essential steps in a risk assessment and management framework. The highest potential risk for human exposure to NOs occurs in the workplace [4]. NOs are stored and handled in workplaces that span from research to production, usage and applications in different processes [5-7]. NOs are also unintentionally formed in various work places as Viitanen et al. [8] have shown with a literature review of 72 publications. As with all materials, 
validated control and monitoring of workplace exposure must be implemented and verified to protect the workforce [9]. A three-tiered approach has been described for occupational exposure assessment [9], which can be part of a risk mitigation strategy. Briefly, Tier I gathers information about process, materials and exposure to determine whether additional assessment is required. Tier II conducts a basic exposure or release assessment using a straightforward approach with easy-to-use portable equipment for release-related investigations and for real-time monitoring. Tier III obtains diverse information on airborne NOs to (a) determine whether exposure has the potential to occur, (b) quantify the level of exposure, and (c) determine the need for additional risk management steps.

Field Exposure Monitoring (FEM) campaigns produce a significant amount of data that are valuable for emissions, exposure and, subsequently, risk assessment. FEM data can also provide insights into the factors affecting process emissions, exposure levels and risks. These insights can be used to quantify the safe conditions of storage and use of NOs and identify efficient risk control techniques [10]. FEM data can be divided into three main categories related to process and material parameters, environmental conditions and worker behavior. Properly collected exposure data can be further refined with mechanistic models to estimate process emissions, emission control efficacies and dispersion of pollutants, along with other exposure determinants depending on the type of measurements. Quantifying emissions according to the process parametrization makes it possible to predict the process impact on work area concentration under different operational conditions. This allows conditions of use for any work scenario to be set by using a probabilistic exposure model [10]. This is currently the most promising approach for setting evidence-based conditions of use for processes and materials in the supply chain, which is one of the objectives in the update of REACH chemical legislation (DG ENV B2, DG GROW F1 “D. Evidence Base, Data collection and Better Regulation Instruments") [11]. However, results from FEM studies are often not comparable to each other due to different methods and metrics [4], as a measurement standardization was recently developed for NO inhalation exposure assessment (CEN EN 17058:2018). The measurement metric (number, surface area, mass) by which the limit should be quantified is unclear specifically because exposure limits are not established for the majority of NOs. The fraction of background particles associated with the NO concentration is usually not well-known and it is ambiguous which parameters are best associated with biological responses [12]. Those uncertainties result in limited and heterogeneous formats of data reporting, which hinders the comparison, validation or integration of data $[4,8,13]$. High-quality data from exposure studies are needed to understand the exposure determinants, which are critical for occupational exposure model development [14]. While developing our template for the ASINA project, the GRACIOUS project (EU H2020, EC-GA No.760840) released templates for release, fate and exposure data collection [15]. Although sharing a number of concepts and determinants, some key differences will be noted in the discussion part.

Data are rarely leveraged beyond their original intended purpose, not only in academia, but in industry as well [16]. Fundamental enablers of digital transformation are technologies that deliver data that adhere to the FAIR principles [17]. FAIR data are considered key components of the new EU Industrial Strategy, Chemicals Strategy for Sustainability and Circular Economy Action Plan driven by the EU Green Deal approach [18], aiming to foster the Safe (and Sustainable) by Design approaches and allow for safe and sustainable innovation [18,19]. FAIR principles were coined in 2014 as a set of minimal guiding rules and practices for research data stewardship in the life sciences [20]. The European Union has released FAIR data management guidelines for Horizon 2020 projects [21] and any Horizon 2020 project that produces, assembles, or processes research data should provide the Data Management Plan (DMP) as an essential deliverable. As an overall practice, data management is connected with the entire lifecycle of data implementation, including the primary steps of data creation, capture, variations and final storage [22]. DMPs facilitate the above aspects, as they play a major role in data FAIRification. As part of making research data FAIR, a DMP should consist of information on: 
- The handling of research data during and after the end of the project;

- What data will be collected, processed and/or generated;

- Which methodology and standards will be applied;

- Whether data will be shared/made open access;

- How data will be curated and preserved.

Previous articles have gone into great detail about FAIR's four higher principles $[17,20,23,24]$. FAIR data principles have been developed to define good practices in data management. What constitutes "good" data management or stewardship is, however, still not clearly defined, and is generally left as a decision for the data creator and/or owner $[23,25]$ and for a specific field of study. Recently, Papadiamantis et al. [26] have defined and proposed the scientific FAIR principles to complement the initial FAIR principles, and assist data creators on the steps needed to capture their data in a FAIR manner as they are produced.

In this paper, we describe the creation of a template for managing field monitoring data by following the execution of the proposed framework, describing the steps taken. We reveal the variables' selection, which serves the FAIRification of exposure monitoring data and facilitates the evaluation of exposure determinants through modelling. Due to numerous combinations of processes and work environments, there are no individual measurement protocols applicable for all conditions. Thus, the focus is on the most important elements of field campaigns and minimum data requirements that data creators should report, which also enables data analysts to validate and reuse them. We show how the new role of data shepherd (see: [26] for shepherd's definition) is involved across diverse fields with the goal to capture data in a reliable and comprehensive way. We describe the inner communication process between stakeholders, which allowed the generation of a co-created template, demonstrating the importance of involvement of all related partners, including data management. The main actors and their responsibilities related to data management are also defined. The template assists the integration of studies, the comparison of working environments and process-related descriptors. The template and guidance are beneficial for exposure assessors to produce and register relevant exposure data by following the FAIR and scientific FAIR principles. This helps researchers and the industrial community to capture relevant information for nanomanufacturing processes. The reporting guidance also helps the professionals involved in the data management process by showing a realistic pathway to template creation and data curation. The paper enriches the scientific community with valuable practical knowledge. The article is also beneficial for data shepherds; since this profession is still new, such detailed works help it to evolve in the right direction.

\section{Materials and Methods}

In the initial article published in the series of data shepherding in nanotechnology, Furxhi et al. [27] visualized the roadmap towards the creation of case-specific data capturing templates for FAIRification purposes, (see Figure 1) in the Horizon 2020 (H2020) ASINA project, with the guidance and support from the Transnational Access program of the H2020 NanoCommons project. The authors identified data cases, acquiring a broad knowledge of data generation and the flow within to meet project goals. Several data will become available from different partners (for data pillars in ASINA, see the aforementioned article).

In this paper, we demonstrate the progress from the proposed framework into the actual realization (template creation), focusing on FEM measurements, with the help and guidance of the data shepherd. Here, the objective of data collection is for logging conditions of use for occupational processes regarding synthesizing, handling or formulating nanostructured materials in the ASINA supply chain. The manufacturers handling and formulating these materials were identified and an initial questionnaire was distributed among the stakeholders. We show how we move from the questionnaires of initial data description circulated among the data creators, (see Section 3.1) to the usage of the preliminary template (see Section 3.2). We demonstrate the results of the inner communication process among partners regarding 
minimum data requirements and the selection of variables that led to a consensus within the project, and a template co-created with all stakeholders (see Section 3.3). In addition, we annotated the dataset to increase interoperability (see Section 3.4).

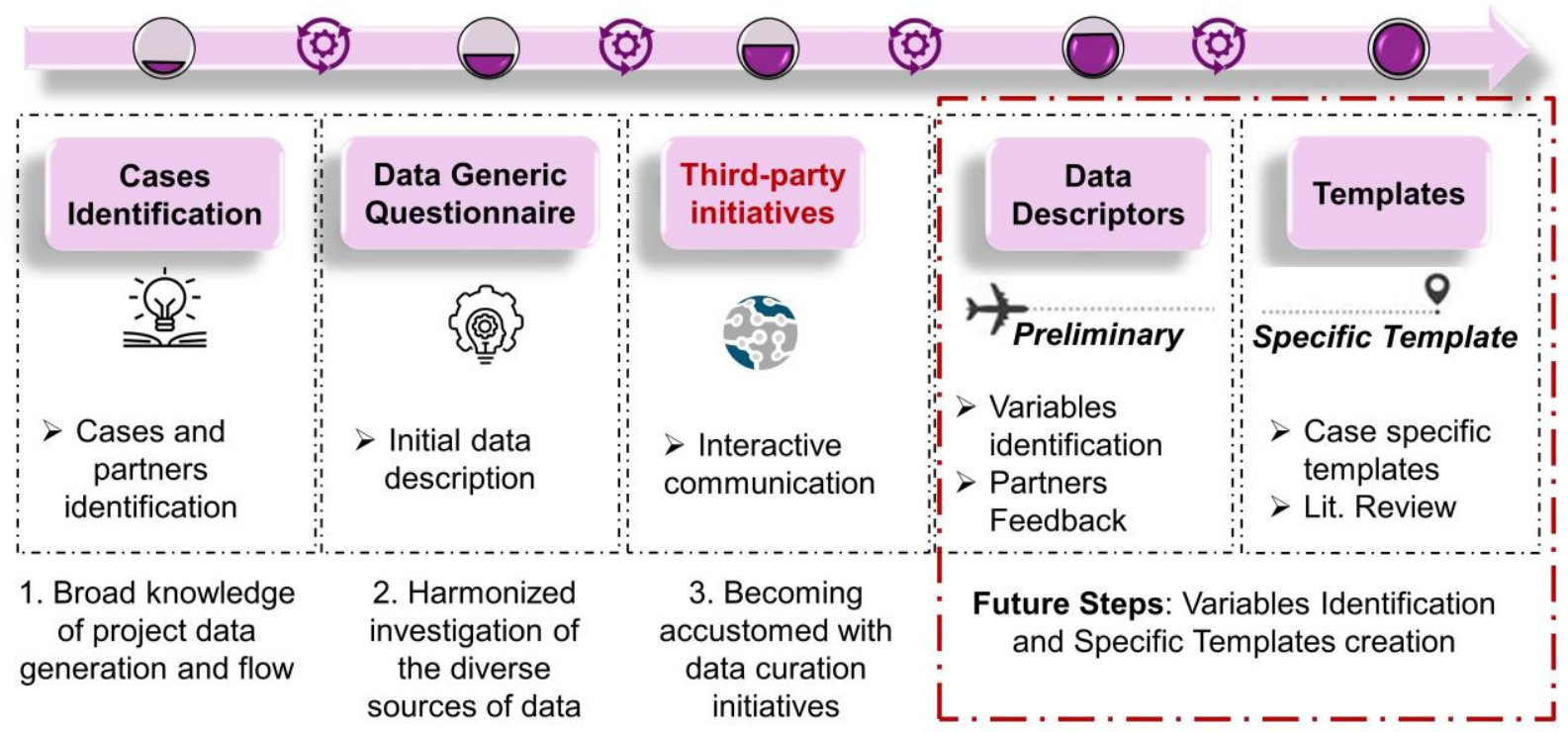

Figure 1. The roadmap towards the creation of case-specific data capturing templates for FAIRification purposes. In this article, we focus on the last two steps (red box) implemented for the field campaign exposure template. A detailed explanation of the previous steps can be found in the aforementioned article.

\section{Results}

\subsection{Initial Data Description-Questionnaire}

A questionnaire to provide a description of the data to be generated is circulated among data creators and analysts. The questionnaire aids the generation of preliminary templates by providing an initial representation of the data to be captured. The responses of data creators and analysts regarding FEM measurements are provided in Table 1 . The questionnaire helps the data shepherd to structure and guide the communication between data creators and relevant involved stakeholders, to identify partners' responsibilities, objectives and expected outcomes, and transfers an overview of needed resources.

The responses from data creators require information regarding the physicochemical characteristics of the NO and their amounts, pilot plants and room characteristics. The outcomes are derived from sampling stations using diverse instruments and monitors to obtain real-time particle number (and distribution), and sampled filters for offline analysis. Data analysts will employ probabilistic exposure models to measurement data for exposure determinants' identification and quantification. Thus, in addition to experimental data, modelling data requirements are implied here but not yet explicitly mentioned. Whether and how measurements of concentration levels at relevant locations will discriminate between sources (background, process or other indoor sources) are not captured at this stage. 
Table 1. Form for collection of information on each dataset from the relevant partners.

\begin{tabular}{|c|c|c|}
\hline \multicolumn{3}{|c|}{ Field Monitoring Campaigns for Exposure Assessment in Pilot Plants } \\
\hline Element & Response-Data Creators & Response-Data Analysts \\
\hline \multicolumn{3}{|c|}{ Data Identification } \\
\hline Dataset description & $\begin{array}{l}\text { Assessment of process emission rates and setting } \\
\text { conditions of use. }\end{array}$ & $\begin{array}{l}\text { Design field monitoring campaigns to } \\
\text { quantify exposure determinants and } \\
\text { workers exposure. }\end{array}$ \\
\hline Source & Experimental measurement data. & Computational data \\
\hline \multicolumn{3}{|c|}{ Partner's activities and responsibilities } \\
\hline $\begin{array}{l}\text { Partner owner of the data; copyright } \\
\text { holder }\end{array}$ & $\begin{array}{l}\text { Each partner is responsible for the data provided } \\
\text { to the data set. }\end{array}$ & $\begin{array}{l}\text { Each partner is responsible for the } \\
\text { data provided to the data set. }\end{array}$ \\
\hline $\begin{array}{l}\text { Partner in charge of data } \\
\text { collection/analysis/storage/related } \\
\text { WP (s) }\end{array}$ & $\begin{array}{l}\text { Data collection: Data creators and Shepherd. } \\
\text { Development of measurement matrix with } \\
\text { stakeholders and reporting plan. }\end{array}$ & $\begin{array}{l}\text { Data analysis: Data analyst, Data } \\
\text { creators and Shepherd. Bridging } \\
\text { information. }\end{array}$ \\
\hline
\end{tabular}

Description of the information required (WPs and/or Tasks) in order to move forward.
-NOs used in the processes and their use amounts per task/shift.

-Pilot plants characteristics: dimensions, aspiration hood (position, size, flow rate).
Design of the measurement's matrix with contextual information.

\section{Expected outcomes}

-Particle concentration.

-Particle size distribution.

-Samples for off-line SEM particle characterization.
-Quantification of workstation concentrations, exposure levels, and other relevant exposure determinants.

measurement variables/outcomes.

\section{Standards}

Set up of an experimental sampling station composed of:

-SMPS to obtain the particle size distribution from $0.01 \mu \mathrm{m}$ to $0.7 \mu \mathrm{m}$.

Detailed description of the methods/protocols
-Optical Particle Counter to obtain the particle size distribution from $0.3 \mu \mathrm{m}$ to $20 \mu \mathrm{m}$. -Monitors to obtain the LDSA (Partector-Naneos and MiniDisc-Testo)

-Total filter samplers.

Measurement standards (e.g., CEN EN 17058:2018; EN689)

\author{
-Analysis of mass flows, process \\ emission rates and other relevant \\ exposure determinants. \\ -Validate mechanistic model against \\ personal exposure. \\ -Apply probabilistic model to \\ evaluate exposure. \\ -Set conditions of use.
}

\subsection{Preliminary Template from NIKC}

NanoCommons' suggestions for data capture include the CEINT NanoInformatics Knowledge Commons (NIKC) Excel spreadsheet [28]. The CEINT-NIKC template was modified (simplified and streamlined via the H2020 NanoCommons infrastructure project) for use by the H2020 project NanoFASE, to capture complex mesocosm experimental data. Spreadsheets are a common format used by most professionals of all subfields of science and, due to their simplicity and familiarity, are a reasonable choice for data entry format. For information related to the template, please refer to [27].

In this article, we focus on the Method and Measurement Tabs (Figure 2). The other tabs of the worksheet (see Supplementary Materials, Template) contain referencing information related to data creators (People and Institution), or information related to any publication where data have been used (Publication). The Method Tabs contain Protocol and Instrument tabs that capture knowledge regarding the instrumentation and protocols related to sample preparation for offline characterization analysis when relevant. The Measurement tab is where information (measurement matrix) related to the process, environmental descriptors, work shift information, $\mathrm{NO}$ and matrix is captured. The main data 
recorded are location-based time series of airborne measurements performed during the manufacturing process. Finally, the Dictionary tab contains a detailed schematic metadata description and ontological annotation of the descriptors.

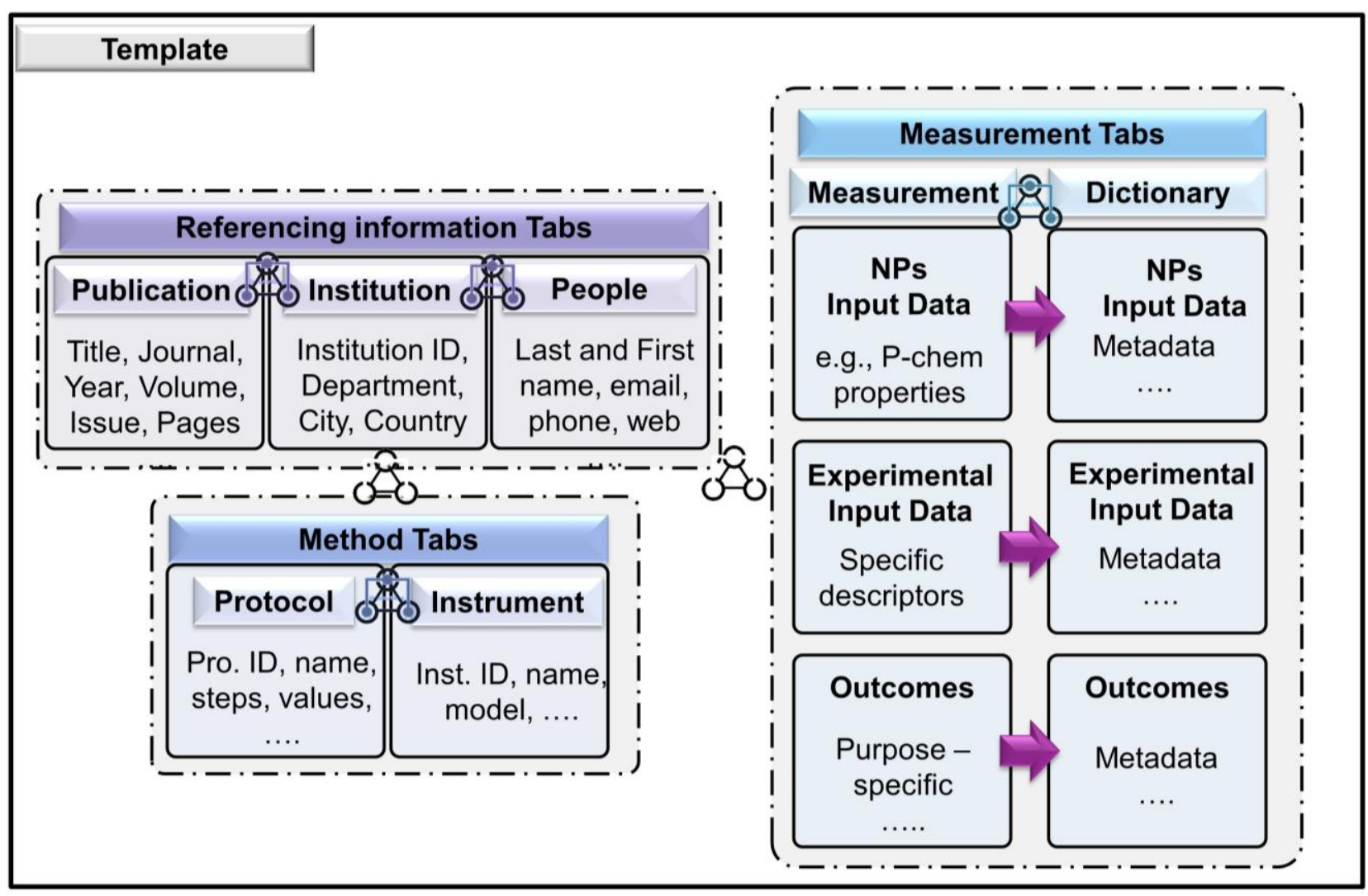

Figure 2. Dataset visualization. Tabs and variables included in each main tab.

\subsection{Literature, Inner-Communication and Descriptors Identification}

Having at hand the data outline from the questionnaires and the preliminary NIKC template, a literature search and multiple experts' feedback are essential in order to merge the two sources in revealing the present variables of importance while maintaining simplicity. This step is the most challenging part in the DMP and FAIRification processes, where the data shepherd and the stakeholders acquire spherical knowledge in the multidisciplinary fields of exposure assessment and modelling. The variables' significance is defined based on stakeholder's expertise and needs to reach project-specific goals. For example, particle size distribution can be applied to calculate the deposited dose during inhalation and to estimate particle deposition losses onto workplace surfaces and filter filtration efficiency. For predictive exposure assessment, particle emission rate from the chamber to the room is a requirement entailing information of air flow rates, besides the concentration measurements. Predictive exposure assessment requires knowledge of the exposure determinants, while source characterization requires measurements of concentration according to the process parametrization and analysis of mass flows [10]. Data availability and reliability following a tiered approach defines the precision of the modelled emission rates. Limited data quality results in precautionary emission rate estimates, higher exposure estimates and more stringent conditions of use. Data reporting requirements (Tier II/III) for data creators for each assessment [9] include information on emission sources, confounding factors, record of workplace activities (see Sections 3.3.1 and 3.3.2), time series of workplace concentrations linked to the process, background concentrations and off-line analyses for chemical and/or morphological information (see Section 3.3.4) and information regarding instrumentation (see Section 3.3.5). To visualize the above information that the template 
captures, we compile the requirements for a demonstration scenario for spray coating machinery (Figure 3).

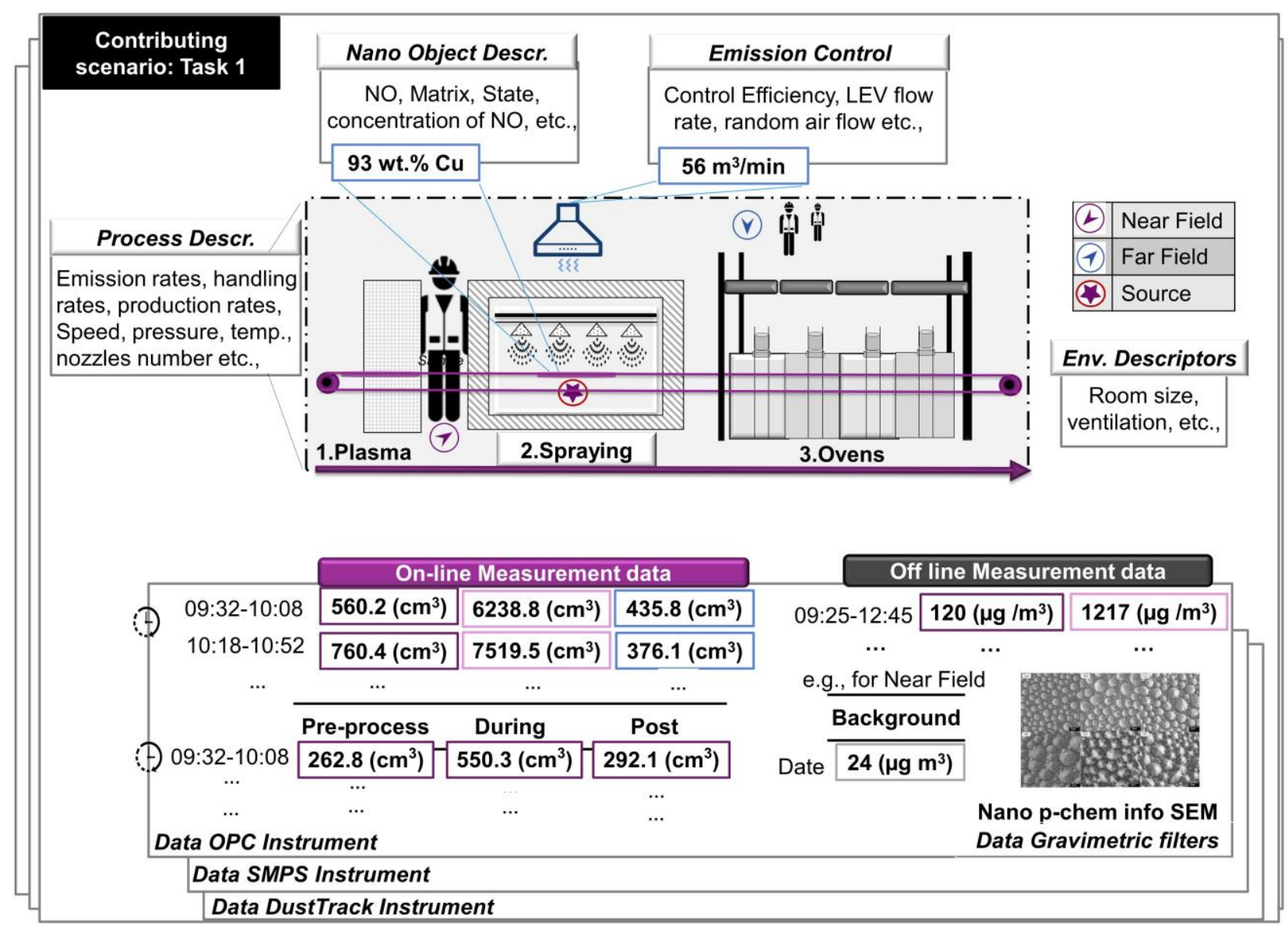

Figure 3. Visualization of exposure and emission data generation. The figure demonstrates a snapshot of data that are generated in one process, specifically one contributing scenario of an extensive exposure assessment.

\subsubsection{Work Shift and Process-Related Descriptors}

A comprehensive FEM requires the quantification of exposure along the process and work-related time intervals [3], which requires process-specific concentrations, e.g., [29,30]. In order to do this, work shift descriptors are captured to describe the contributing scenario in a task-specific manner. In cases where the production process is continuous, this information is less relevant (Table 2). Process definition is used to capture the general identification of a process using variable terminology in alignment with ECHA R.14 [31] requirements for exposure assessment, e.g., Life Cycle Stage (LCS), Sector of use (SU). ECHA descriptors are limited to main industrial process categories and may not always reflect the scenario for NO exposure scenarios. Therefore, we used ECHA descriptors selectively and propose, along with the workplace observations, a visualization (sketch, technical drawing, photo) as an essential aid in comprehensibly demonstrating the entire process and scenario to the data users (see Supplementary Materials, Measurement Tab). 
Table 2. Work shift descriptors and process definition.

\begin{tabular}{|c|c|c|c|}
\hline Category & Variables & Metrics & Metadata Description \\
\hline \multirow{5}{*}{$\begin{array}{l}\text { Work Shift } \\
\text { Descriptors }\end{array}$} & Task Identifier & Text & Short identifier, e.g., Task 1 \\
\hline & Use/Task name & Text & Task name, e.g., Powder pouring \\
\hline & Description & Text & $\begin{array}{l}\text { Task descriptions linked to process descriptors. If } \\
\text { sampling is performed over multiple tasks each task } \\
\text { should be described separately, e.g., Task } 1.1 \text { and } 1.2\end{array}$ \\
\hline & Task duration & [min] & Task duration \\
\hline & Time spent near process & [min] & $\begin{array}{l}\text { Specify what is considered as near the source. For } \\
\text { example, near the source is when distance is }<2 \mathrm{~m} \text {, for } \\
\text { point emission sources and }<10 \mathrm{~m} \text { for diffusive sources } \\
\text { (well mixed concentrations). The distances here are based } \\
\text { on authors decisions. }\end{array}$ \\
\hline \multirow{5}{*}{$\begin{array}{c}\text { Process } \\
\text { Definition }\end{array}$} & Process Diagram & $\begin{array}{c}\text { Picture (sketches, } \\
\text { photos) }\end{array}$ & $\begin{array}{l}\text { Process visualization including identified exposure } \\
\text { determinants, such as source, process closures, LEVs and } \\
\text { worker location. }\end{array}$ \\
\hline & Life Cycle Stage (LCS) & List & LCS code according to ECHA use descriptor \\
\hline & Sector of use (SU) & List & SU code according to ECHA use descriptor \\
\hline & Process category (PROC) & List & PROC code according to ECHA use descriptor \\
\hline & Description & Text & $\begin{array}{l}\text { General description of the process, equipment, relevant } \\
\text { process parameters, material chemical compositions and } \\
\text { form (solid, liquid, gas). It is recommended to provide } \\
\text { process machine manufacturer and model and material } \\
\text { safety data sheets. }\end{array}$ \\
\hline
\end{tabular}

Generic process descriptors such as automation, duration, emission rates (if available) and production rates are needed to estimate exposure times, emissions according to process parameters, production volumes (work intensity) and dispersion of the pollutants at the workstation (Table 3). ASINA specifically includes diverse processes (synthesis, spray coating, microemulsion, spray freeze drying, dip coating and padding, screen printing and cosmetic formulation) that have to be captured. Process parameters capture the variables that can affect the emission rate or dispersion of pollutants. Primary factors, such as process temperature, speed and pressure, are generic and should be included. The user can clarify the speed (roll speed, agitation speed of the emulsifying process) or temperature (fluctuation, or drying temperature during thermal curing) of relevance to be included. Secondary factors, which are process-specific, for example, in spray processes (Figure 3), information such as the number, arrangement and type of nozzles should be reported. Plasma treatment, on the other hand, is defined by the type and flow of treatment gas and the electrode type (ceramic or metallic). Those factors are inserted by the user according to the specific case. An example of data generated and capturing in this scenario are included in the Supplementary Materials. 
Table 3. Generic process descriptors captured in the template.

\begin{tabular}{|c|c|c|c|}
\hline Category & Variables & Metric & Metadata Description \\
\hline & Process parameters & Text & $\begin{array}{l}\text { Factors that have an effect on the process emissions } \\
\text { including materials. This is relevant for other users so that } \\
\text { they can apply the process emissions reliably for their case. } \\
\text { Data creators and partners are responsible for identifying } \\
\text { relevant process parameters affecting the emission. Koivisto } \\
\text { et al. [32] show some examples of relevant process } \\
\text { parameters for specific processes and materials. } \\
\text { Example: Temperature, speed, pressure. The user has the } \\
\text { freedom to insert process parameters relevant to the case. }\end{array}$ \\
\hline \multirow[t]{5}{*}{$\begin{array}{l}\text { Generic Process } \\
\text { Descriptors }\end{array}$} & Level of automation & List & $\begin{array}{l}\text { If workers' time use is not known, the level of process } \\
\text { automation can be used to estimate the worker exposure } \\
\text { time as: Fully-Workers are only involved in supervision } \\
\text { and control walks. Worker exposure time }<30 \text { min during } \\
\text { an } 8 \text { h work shift. Highly-Very limited manual invention is } \\
\text { required to run. Contact with the substance may be possible } \\
\text { for a very limited duration. Worker exposure time between } \\
30 \text { and } 90 \text { min during an } 8 \mathrm{~h} \text { work shift. Semi-Manual } \\
\text { intervention is repeatedly required, although large parts of } \\
\text { the process are machinery assisted. Worker exposure time } \\
\text { between } 90 \text { and } 240 \text { min during an } 8 \text { h work shift. } \\
\text { Manual-This process requires an operator, who is } \\
\text { conducting a task manually. Worker exposure time is over } \\
240 \text { min during an } 8 \text { h work shift. The time intervals here } \\
\text { are based on authors' selections. }\end{array}$ \\
\hline & Process duration & [min] & $\begin{array}{l}\text { Equal or smaller than task duration, depending on if the } \\
\text { process is continuous or not. Process duration and rate } \\
\text { should equal to the production amount during work shift. }\end{array}$ \\
\hline & $\begin{array}{l}\text { Material process } \\
\text { /handling rate }\end{array}$ & [e.g., kg/min] & $\begin{array}{l}\text { This describes the intensity of process during the task and } \\
\text { should be expressed in units, such as: } \\
\text { - Material transfer, handling or synthesis (e.g., } \mathrm{kg} / \mathrm{min} \text {; } \\
\mathrm{m}^{3} / \mathrm{min} \text { ) } \\
\left.\text { - Surface treatment (e.g., } \mathrm{m}^{2} / \mathrm{min}\right) \\
\text { - Component production or processing (e.g., pieces } / \mathrm{min} \text {, } \\
\mathrm{m}^{2} / \mathrm{min} \text { ) }\end{array}$ \\
\hline & $\begin{array}{l}\text { Process emission } \\
\text { rate/factor }\end{array}$ & [e.g., $\mu \mathrm{g} / \mathrm{min}]$ & $\begin{array}{l}\text { Process emission described in scalable quantities to } \\
\text { calculate emission in different exposure scenarios. For } \\
\text { example, emission factor can be expressed for surface } \\
\text { treatment as } \mathrm{mg} / \mathrm{m}^{2} \text {, material removal/additive processes } \\
\text { as } \mathrm{mg} / \mathrm{kg} \text {, component production as } \mathrm{mg} / \text { component, } \\
\text { welding as } \mathrm{mg} / \mathrm{cm} \text { of welding, etc. }\end{array}$ \\
\hline & Production Rates & $\begin{array}{l}\text { [e.g., } \mathrm{m}^{2} / \mathrm{min} \text {, } \\
\text { pieces } / \mathrm{min}]\end{array}$ & $\begin{array}{l}\text { Similarly, as for emission rate, the production rate is } \\
\text { described in scalable quantities. }\end{array}$ \\
\hline
\end{tabular}

\subsubsection{Emission Control and Environmental Descriptors}

Emission control descriptors of the machinery are included in the template (Table 4). Local exhaust ventilation (LEV) efficiency plays an important role for concentration near the source when LEV is on. LEV can be designed so that it captures a fraction of emissions before entering the process room (e.g., fume hoods with $95 \%$ capturing efficiency). Measuring LEV concentration levels, extraction volume flow and filtration efficiency allow quantification of emissions to outdoor air or room air in cases of circulating LEV systems, where the air is returned to the room. The fraction that is escaped to the room can be specified from near field concentration and mixing of the concentration to the rest of the room by using balance principles. 
Table 4. Emission control descriptors.

\begin{tabular}{|c|c|c|c|}
\hline Category & Variables & Metric & Metadata Description \\
\hline \multirow[t]{3}{*}{$\begin{array}{l}\text { Emission Control } \\
\text { descriptors in process } \\
\text { machine }\end{array}$} & $\begin{array}{l}\text { Emission control } \\
\text { type }\end{array}$ & List & $\begin{array}{l}\text { It is recommended to report the distance from the source and } \\
\text { extraction funnel surface area for LEV and, for closures, the open } \\
\text { surface area to evaluate LEV capturing efficiency (e.g., } \\
\text { https: / /www.hse.gov.uk/lev/ calculator.htm (accessed on } 8 \text { June } \\
\text { 2021)). Emission control categorization helps to describe control } \\
\text { techniques [33,34]: } 1 \text {. Enclosure (automation): physical } \\
\text { containment or enclosure of the source of emission; } 2 \text {. LEV: } \\
\text { exhaust ventilation systems located in close proximity of and } \\
\text { directed at the source of emission; } 3 \text {. Specialized ventilation } \\
\text { systems: mechanical ventilation systems specifically designed for } \\
\text { displacement of air contaminants in small, designated areas or } \\
\text { systems intended to supply fresh air to workers; } 4 \text {. Suppression } \\
\text { techniques: techniques where an additive is added to an activity } \\
\text { or process in an attempt to suppress emissions; } 5 \text {. Segregation } \\
\text { sources: isolation or segregation of sources from the work } \\
\text { environment without containment of the emission source itself; } 6 \text {. } \\
\text { Worker separation: a personal enclosure within a work } \\
\text { environment. It is recommended to provide the emission } \\
\text { control's manufacturer, model and efficiency. Note that emission } \\
\text { control efficacies should be reported as well and in worker } \\
\text { separation, the time that the worker spends in a separated area } \\
\text { and close to the process. }\end{array}$ \\
\hline & $\begin{array}{l}\text { Local Control } \\
\text { Efficiency }\end{array}$ & {$[\%]$} & $\begin{array}{l}\text { Efficiency is } 100 \% \text { for perfect local control capturing all emissions } \\
\text { and } 0 \% \text { when it acts as a ventilation exhaust near the source. }\end{array}$ \\
\hline & LEV flow rate & {$\left[\mathrm{m}^{3} / \mathrm{min}\right]$} & $\begin{array}{l}\text { LEV air extraction and exhaust locations should be specified (e.g., } \\
\text { mechanically assisted LEV where replacement air is partially } \\
\text { from outdoors or LEV circulating the room air through a filter). }\end{array}$ \\
\hline
\end{tabular}

Environmental descriptors are needed for the data analyst to estimate dilution and removal of pollutants from the process and other potential sources (Table 5). Separating process emissions from background emissions enables the calculation of process-specific exposure levels. Random air speed is a critical factor defining the mixing of emissions from near field to the rest of the room that can be measured by using, e.g., a hot wire anemometer. The random air velocity can be modified by process fans and temperature differences between process and room air and environmental conditions.

\subsubsection{Nanostructured Materials Descriptors}

Data regarding the NOs used during manufacturing are required for exposure and hazard assessment. More importantly, this information enables data integration and traceability across the stages (NOs synthesis $\rightarrow$ incorporation to matrix $\rightarrow$ nanostructured materials) and is required for human and environmental hazard assessment [32]. Nanostructured materials descriptors (Table 6) are inspired by the particle emission library for articles and products containing NO [32], capturing information such as the category of the product in which the $\mathrm{NO}$ is incorporated, a description of the substrate, the NO used in the process (name, ID, core information), external and internal layer, the provider (partners within the project or external provider), and concentration in the matrix (e.g., $\mathrm{mg}$ of $\mathrm{TiO}_{2}$ per $\mathrm{m}^{2}$ of textiles). The form of NO in the matrix (embedded into the matrix, surface-bound, incorporated, impregnated) can be used to assess potential NO release in different life cycle stages.

\subsubsection{Time-Series Measurements}

Measurements can be divided into on-line (Table 7) and off-line (Table 8) characterization. Concentration data are based on location, such as Breathing Zone (BZ), Near Field (NF), Far Field (FF), source, ventilation and process phase (post, during, pre). Those can be used to quantify the NO concentration where background (BG) concentrations from 
natural and incidental sources are subtracted [4]. Two common approaches to specify NO concentration levels are to 1 ) subtract pre- and post-process concentrations from process concentration levels or 2) measuring simultaneously at a co-location not influenced by the investigated process emissions and subtract the concentration from process concentration level [9]. Method 1 assumes that the BG concentration level is constant during the process and method 2 assumes that the co-location concentration represents the workstation location's ventilation air concentration.

Table 5. Environmental descriptors.

\begin{tabular}{|c|c|c|c|}
\hline Category & Variables & Metric & Metadata Description \\
\hline \multirow{5}{*}{$\begin{array}{l}\text { Environmental } \\
\text { descriptors }\end{array}$} & Room size & {$\left[\mathrm{m}^{3}\right]$} & $\begin{array}{l}\text { An approximate of the room volume where air exchange is } \\
\text { considered to follow the room ventilation rate. }\end{array}$ \\
\hline & Room ventilation & {$\left[\mathrm{m}^{3} / \mathrm{min}\right.$ or $\left.1 / \mathrm{h}\right]$} & $\begin{array}{l}\text { Ventilation by LEV should be treated separately from the } \\
\text { general ventilation. }\end{array}$ \\
\hline & $\begin{array}{l}\text { Other possible } \\
\text { emissions sources }\end{array}$ & Text & $\begin{array}{l}\text { Emissions from other processes in the same room as the } \\
\text { sampler can increase the concentration level. Incoming } \\
\text { ventilation air may also transport pollutants depending on } \\
\text { the ventilation design and location of the facility. All active } \\
\text { processes during the task that could impact measurements } \\
\text { should be mentioned. }\end{array}$ \\
\hline & $\begin{array}{l}\text { Workstation } \\
\text { closure }\end{array}$ & Text & $\begin{array}{l}\text { The workstation closure where the worker performs the } \\
\text { task is used to estimate how much air mixing between near } \\
\text { and far field is limited. This is relevant mainly for point } \\
\text { sources. Workstation closure is expressed as a side of a } \\
\text { cube/rectangle, where sides may be closed in combination } \\
\text { of back, one side, both sides and top. }\end{array}$ \\
\hline & $\begin{array}{l}\text { Random Air flow } \\
\text { at workstation }\end{array}$ & {$[\mathrm{m} / \mathrm{min}]$} & $\begin{array}{l}\text { Random air speed gives information about pollution } \\
\text { dispersion near the source [35]. Near is defined as the } \\
\text { distance within the worker breathing zone and the source. } \\
\text { Typically, this varies from } 30 \mathrm{~cm} \text { to } 1 \mathrm{~m} \text {. }\end{array}$ \\
\hline
\end{tabular}

Table 6. Nanostructured material descriptors.

\begin{tabular}{|c|c|c|c|}
\hline Category & Variables & Metric & Metadata Description \\
\hline \multirow{8}{*}{$\begin{array}{c}\text { Nanostructured } \\
\text { material Descriptors }\end{array}$} & $\begin{array}{l}\text { Article Category (AC) } \\
\text { and sub-category }\end{array}$ & List & AC code according to ECHA use descriptors \\
\hline & Substrate (Matrix type) & Text & $\begin{array}{l}\text { Description of the matrix (Polyester fibers, } \\
\text { Polymethylmethacrylate, clay, epoxy, fil, liquid) and chemical } \\
\text { composition }\end{array}$ \\
\hline & NO name and core & Text & $\begin{array}{l}\text { Name, ID code (i.e., JRCxxx) CAS and or ES number and } \\
\text { chemical composition, i.e., TiO2 pigment ( } 93 \% \text { rutile), (Tioxid } \\
\text { TR81; CAS-Nr. 13463-67-7) }\end{array}$ \\
\hline & External Layer & Text & NO name in surface layer 1 (more external layer) \\
\hline & Inner Layer & Text & NO name in surface layer 2 (inner layer) \\
\hline & NO Provider/Supplier & Text & $\begin{array}{l}\text { NO manufacturer provider (partner related to the project } \\
\text { ASINA) or supplier }\end{array}$ \\
\hline & Concentration & [wt. \%] & $\begin{array}{l}\text { NO concentration in material mass unit, e.g., wt. } \%, \mu \mathrm{g} / \mathrm{g} \text {, etc. } \\
\text { Usually, this information is provided by a different partner, but } \\
\text { the final concentration of NOs in a product is of paramount } \\
\text { importance and should be captured when known. }\end{array}$ \\
\hline & Form of $\mathrm{NO}$ in matrix & Text & $\begin{array}{l}\text { State of NO (s) in the matrix/media: embedded into, surface } \\
\text { bound, incorporated, impregnated, in liquid, dispersion, } \\
\text { powder, solid, paste }\end{array}$ \\
\hline
\end{tabular}


Table 7. On-line Measurement's descriptors.

\begin{tabular}{|c|c|c|c|}
\hline Category & Variables & Metrics & Metadata Description \\
\hline \multirow{11}{*}{$\begin{array}{l}\text { On-line } \\
\text { Measurements } \\
\text { based on Location } \\
\text { and Phase }\end{array}$} & $\begin{array}{l}\text { Collection } \\
\text { Interval }\end{array}$ & hh:mm-hh:mm & Start-End Time of the measurement \\
\hline & Instrument & & $\begin{array}{l}\text { Instrument name. Detailed information regarding instruments } \\
\text { is inserted in a different tab (Instrument Tab) }\end{array}$ \\
\hline & Ventilation & {$\left[\mathrm{m}^{3} / \mathrm{min}\right.$ or $\left.1 / \mathrm{h}\right]$} & Incoming/outgoing ventilation air \\
\hline & Source & \multirow{8}{*}{$\begin{array}{c}\text { Metric: } \\
\text { P Numb }\left(\mathrm{cm}^{-3}\right), \\
\text { Lung Deposited } \\
\left(\mu \mathrm{m}^{2} \mathrm{~cm}^{-3}\right), \text { Size } \\
\text { Distribution }\left(\mathrm{dN} / \mathrm{d}\left(\mathrm{D}_{\mathrm{p}}\right)\right) \text {, } \\
\text { Mass }\left(\mu \mathrm{g} \mathrm{m}^{-3}\right) \\
\text { AND } \\
\text { Variations: Average, } \\
\text { Geometric mean }(\mathrm{GM}), \\
\text { Geometric standard } \\
\text { deviation }(\mathrm{GSD}), \\
\text { Arithmetic mean }(\mathrm{AM}), \\
\text { Standard deviation }(\mathrm{SD})\end{array}$} & $\begin{array}{l}\text { Very close to the source or at the source (e.g., tailpipe, inside the } \\
\text { process closure or inside fume chamber). } \\
\text { Report average and standard deviation }\end{array}$ \\
\hline & NF & & $\begin{array}{l}\text { NF-Close to the source where pollution is mixed to the rest of } \\
\text { the room. At working station, NF should cover the worker's } \\
\text { breathing zone and source, and provide the distance from the } \\
\text { source. }\end{array}$ \\
\hline & FF & & $\begin{array}{l}\text { FF-Typically } 5 \text { to } 10 \mathrm{~m} \text { from the NF measured in the same } \\
\text { room (same room where the source is). }\end{array}$ \\
\hline & $\mathrm{BZ}$ & & $\begin{array}{l}\text { Breathing zone measured within } 30 \mathrm{~cm} \text { from the worker's nose } \\
\text { and mouth (EN 689) }\end{array}$ \\
\hline & BG & & $\begin{array}{l}\text { Other spaces where ventilation replacement air flows are } \\
\text { potentially carrying pollutants to the process room. } \\
\text { Background can be estimated from pre- and post-process } \\
\text { measurements or by measuring background concentration from } \\
\text { FF that is not affected by the source emissions. }\end{array}$ \\
\hline & Pre-process & & Pre- and post-process concentrations are one way to estimate \\
\hline & $\begin{array}{l}\text { Post-Process } \\
\text { concentra- } \\
\text { tion }\end{array}$ & & $\begin{array}{l}\text { the concentrations from the process. This shows how much the } \\
\text { concentration is increased by the process, when assuming } \\
\text { background concentration as constant (as average of pre- and } \\
\text { post-concentrations). Specified for NF, FF, or source depending } \\
\text { on the assessment objective (e.g., worker or co-worker exposure } \\
\text { to process particles or emissions from the process, respectively); } \\
\text { (2) Specified for any instrument. } \\
\text { Local background particle exposure: local work area eight-hour } \\
\text { time-weighted average particle number or mass concentration } \\
\text { that excludes any contribution of particles from the } \\
\text { nanotechnology process. This value is specific to each work } \\
\text { environment. This value should be determined following } \\
\text { repeated measurement of the particle number and mass } \\
\text { concentration when the nanotechnology process is not in } \\
\text { operation. The results of such measurement should be } \\
\text { converted to an 8-hour time-weighted average value and the } \\
\text { median of all values used as the basis for the recommended } \\
\text { local particle reference value. }\end{array}$ \\
\hline & $\begin{array}{l}\text { Process con- } \\
\text { centration }\end{array}$ & & Concentration during the process. \\
\hline \multirow{5}{*}{$\begin{array}{l}\text { Log-normal size } \\
\text { distribution Descr. }\end{array}$} & Instrument & & \\
\hline & $\begin{array}{l}\text { Location and } \\
\text { Phase }\end{array}$ & & NF, FF, BG, etc., Post-/During/Pre-process \\
\hline & $\mathrm{X}$ & {$\left[\mathrm{N}\left(\mathrm{cm}^{3}\right)\right]$} & P number concentration (>10 nm) \\
\hline & GMD & {$[\mathrm{nm}]$} & $\begin{array}{l}\text { geometric mean diameter, AND information such as: particle } \\
\text { diameter corresponding to } 50 \% \text { of the cumulative distribution }\end{array}$ \\
\hline & GSD & {$[-]$} & $\begin{array}{l}\text { geometric standard deviation, AND information such as: } \\
\text { particle diameter corresponding to } 84 \% \text { of the cumulative }\end{array}$ \\
\hline
\end{tabular}


Table 8. Offline measurements descriptors.

\begin{tabular}{|c|c|c|c|}
\hline Category & Variables & Metrics & Metadata Description \\
\hline \multirow{9}{*}{$\begin{array}{l}\text { Offline } \\
\text { Measurements and } \\
\text { Analysis }\end{array}$} & Collection Interval & hh:mm-hh:mm & \\
\hline & Instrument & Text & $\begin{array}{l}\text { Instrument name. Detailed information } \\
\text { regarding instruments is inserted in a different } \\
\text { tab (Instrument Tab) }\end{array}$ \\
\hline & $\begin{array}{l}\text { Sample collection/ } \\
\text { description }\end{array}$ & Text & $\begin{array}{l}\text { A description of the sample collection, e.g., } \\
\text { gravimetric, surface dust sample }\end{array}$ \\
\hline & Ventilation & {$\left[\mathrm{m}^{3} / \mathrm{min}\right.$ or $\left.1 / \mathrm{h}\right]$} & \\
\hline & Source & Metric: P Numb $\left(\mathrm{cm}^{-3}\right)$ & \multirow{5}{*}{ Same as Table 7} \\
\hline & NF & $\begin{array}{l}\text { Lung Deposited } \\
\left(\mathrm{um}^{2} \mathrm{~cm}^{-3}\right) \text { Size Distri }\end{array}$ & \\
\hline & $\mathrm{FF}$ & $(\mathrm{dN} / \mathrm{d}(\mathrm{dp}))$, Mass $\left(\mu \mathrm{g} \mathrm{m}^{-3}\right)$ & \\
\hline & $\mathrm{BZ}$ & AND & \\
\hline & BG & $\begin{array}{l}\text { AM, GSD, } \\
\end{array}$ & \\
\hline \multirow{4}{*}{$\begin{array}{l}\text { Chemical } \\
\text { Information }\end{array}$} & Instrument & Text & I.e., ICP-MS, EDX \\
\hline & \multirow{3}{*}{\multicolumn{2}{|c|}{$\begin{array}{l}\text { E.g., Elemental composition (wt.\%), Impurities, } \\
\text { Crystallinity phase }\end{array}$}} & $\begin{array}{l}\text { Elemental composition, i.e., Major: Ti, O Minor: } \\
\mathrm{Si}, \mathrm{Al}, \mathrm{Zr}, \mathrm{P} \text {, and organic coating }\end{array}$ \\
\hline & & & $\begin{array}{l}\text { Impurities: Any unwanted organic, inorganic, } \\
\text { and residual solvents in drug substances and } \\
\text { final products. }\end{array}$ \\
\hline & & & $\begin{array}{l}\text { The detection and quantification of the amount } \\
\text { of amorphous material within a highly } \\
\text { crystalline substance. }\end{array}$ \\
\hline \multirow{6}{*}{ StructuralInformation } & Instrument & Text & I.e., SEM, TEM, BET \\
\hline & \multirow{5}{*}{\multicolumn{2}{|c|}{$\begin{array}{l}\text { E.g., Pristine size }(\mathrm{nm}), \text { Shape, Specific surface area } \\
\left(\mathrm{m}^{2} / \mathrm{g}\right) \text {, Bulk density }\left(\mathrm{g} / \mathrm{cm}^{3}\right) \text {, Zeta potential }(\mathrm{mV}), \\
\text { Dissolution rate (mass } / \text { time unit), Solubility }(\mathrm{g} / \mathrm{L})\end{array}$}} & $\begin{array}{l}\text { Specific surface area total surface area of a } \\
\text { material per unit of mass, or solid or bulk } \\
\text { volume }\end{array}$ \\
\hline & & & Bulk density \\
\hline & & & $\begin{array}{l}\text { Zeta: The measurement of the overall charge a } \\
\text { particle acquires in a specific medium }\end{array}$ \\
\hline & & & $\begin{array}{l}\text { Dissolution rate actual release rate of the } \\
\text { compound at the given particle size, etc. }\end{array}$ \\
\hline & & & $\begin{array}{l}\text { Solubility: capacity of a solute to dissolve in a } \\
\text { pure solvent }\end{array}$ \\
\hline
\end{tabular}

The units in the template defined depend on the detection technique. Common on-line particle sizing techniques are light scattering (e.g., optical particle sizer), particle mobility classification and single particle counting (e.g., scanning mobility particle sizer), particle aerodynamic classification and current induced by moving charge particle (e.g., electric low pressure impactor) [29]. It is important to specify the particle sizing technique because it affects the assumptions made during data analysis (e.g., unit conversions; see [29]. On-line personal exposure data are usually based on particles' light scattering and diffusion charging because other portable on-line detection techniques are scarce [36]. From a modelling perspective, particle concentrations at different locations are used to calculate mass flows and further process specific particle emission rates in particle number, surface area, or mass. Furthermore, the concentrations can be exploited to characterize process-specific particle sources and estimate their influence [29].

Particle size distribution information is also added (Table 7), noting that, depending on size distribution and concentration, the deviation in the particle concentration number measured by the different instruments can be up to $50 \%$ [30]. For NO dust collection 
sampling, cassette wall losses should be reported; in conductive cassettes, the wall losses for respirable dust have been reported to be up to $56 \%$ as median wall deposits [37]. Size distribution reporting can be shortened with values of a fitted log-normal distribution that further simplifies the data reporting and applicability. Particle size information can be used for estimating particles' deposition onto surfaces and to calculate the inhalation dose rates of deposited particles. The exposure levels and regional deposited doses during inhalation are used to estimate the inhalation exposure risk of process particle emissions [30].

On-line measurement techniques do not directly differentiate between agglomerates, aggregates and primary particles. Samples for offline analysis is performed to augment real time measurement (Table 8). Results from structural and chemical characterization (p-chem descriptors) of particles discriminate the sources to some degree [36]. Surface area analysis, e.g., by BET, can be used to help differentiate between agglomerates and aggregates [36].

\subsubsection{Instrumentation}

Currently, there is no available instrument capable of meeting all the requirements of exposure characterization of airborne $\mathrm{NO}$ and there is insufficient comparability between measurement methods [38]. A suite of devices is typically used to conduct an exposure characterization [9]; thus, information regarding instruments is included. One tab is dedicated to instrumentation, covering the instrument name, the model, measured metric, detection limit, flow rate, etc., (see Table 9). The instrument is linked to the measurement $\mathrm{tab}$ for each row clarifying the source of the data. The data creator is responsible for correcting potential sampling line diffusion and dilution losses in sampling if not isoaxial and isokinetic sampling and reporting the correction methods.

Table 9. Instrument descriptors.

\begin{tabular}{|c|c|c|}
\hline Category & Variables & Metadata Description \\
\hline \multirow{8}{*}{ Instrument } & Instrument Name & $\begin{array}{l}\text { e.g., FESEX, EDX, ICP-MS, OPC, SMPS, DustTrack, } \\
\text { INSPEC, etc., }\end{array}$ \\
\hline & Model & $\begin{array}{l}\text { e.g., Sigma, DISCMini, Partector, DusttRACK, Bravo, } \\
\text { Pump }\end{array}$ \\
\hline & $\begin{array}{l}\text { Measured Metric } \\
\text { (Outputs) }\end{array}$ & $\begin{array}{l}\text { e.g., Mobility/aerodynamic/optical particle Number } \\
\text { concentration }\left(1 / \mathrm{cm}^{3}\right) \text {, Lung Deposited Surface Area } \\
\left(\mu \mathrm{m}^{2} / \mathrm{cm}^{3}\right) \text {, Particle Size Distribution }(\mathrm{dN} / \mathrm{d}(\mathrm{dp})) \text {, } \\
\text { Particle Mass concentration }\left(\mu \mathrm{g} / \mathrm{m}^{3}\right)\end{array}$ \\
\hline & Detection Technique & $\begin{array}{l}\text { e.g., light scattering, particle mobility classification and } \\
\text { single particle counting, particle aerodynamic } \\
\text { classification and current induced by moving charge } \\
\text { particle }\end{array}$ \\
\hline & Size Range & $\begin{array}{l}\text { e.g., } 30 \mathrm{~nm}-\sim 700 \mathrm{~nm}, 250 \mathrm{~nm}-20 \mu \mathrm{m}, 11.1 \mathrm{~nm}-1082 \mathrm{~nm} \text {, } \\
>30 \mu \mathrm{g}\end{array}$ \\
\hline & Detection Limits & Lower and upper detection limit \\
\hline & Total Flow (L/min) & Instrument sampling flow? \\
\hline & $\begin{array}{l}\text { Manufacturer } \\
\text { Information }\end{array}$ & $\begin{array}{l}\text { e.g., Agilent technology, Santa Clara, USA. TSI, } \\
\text { Shoreview, USA, Grimm Aerodol Technik, Airnring, } \\
\text { Germany. }\end{array}$ \\
\hline
\end{tabular}

Any protocols used during the campaigns are reported in the Protocols tab, i.e., sample preparation techniques of gravimetric assessment, or sample preparation for SEM/TEM analysis. In the case of FEM, any intercomparison results between instruments would be beneficial to be shown. 


\subsection{Annotation}

Metadata are described as data about data and they are required in order for a dataset to be useful to others. Bringing nanodata together in a harmonized and interoperable fashion places demands on the description and curation of data [26]. There are ongoing efforts to encourage the reuse of data facilitated through, e.g., the NanoCommons project or the European Union Observatory for Nanomaterials (EUON) based on the requirements of the European Commission's Open Data policy, which can succeed if complete (data can be found and understood without having to ask the data creators) high-quality data and metadata are available.

In accordance with the European Commission's Open Data Policy and the FAIR data principles, the resultant template (metadata) is semantically annotated using established hierarchically structured domain specific dictionaries-ontologies. By offering a consistent terminology throughout a subject, ontologies are used to facilitate communication between people and organizations [39]. The most used nano-specific ontologies are the NanoParticle Ontology (NPO) and the eNanoMapper (eNM) ontology [26,40]. All descriptors in the measurement and instrument tabs are semantically annotated (see Supplementary Materials, tab Dictionary), to supplement the metadata. The descriptors can be annotated with several ontological terms, allowing the usage of numerous dictionaries to improve interoperability [40]. We combined and integrated different ontological IDs from diverse libraries when a single annotation from a source was missing.

The annotation increases interoperability and facilitates explicit understanding by providing a detailed metadata description to the dataset. In this way, the dataset becomes machine findable and interoperable with analogous datasets and supports indexing of data. The annotation requirements of the computational community are different from those of the regulatory community [26]. The current available data may be interoperable under a regulatory or scientific context but require processing to become computationally usable. Adding a comprehensive description is a key part that enables novel modelling and machine learning applications to be used and/or created by the nanosafety community.

\section{Discussion}

Clark et al. [41] noted that studies lack information on measurement strategy, and to date, establishing harmonized guidance is still pending. Harmonization is challenging because of the high diversity of processing techniques, materials and environmental conditions including emission and exposure controls. Various measurement strategies have been used within national projects, triggering interest in developing a harmonized approach to ensemble usability and comparability $[8,9,36]$. The template presented in this study works as a comprehensive worksheet, allowing users to import information regarding sampling locations and experimental details.

The template combines the information into tabs dedicated to measurements, instruments and protocols, is grounded on diverse sources $[3,9,12-14,31,36]$ and offers an examination of both task-based emissions and airborne levels. Furthermore, the reliability of exposure models depends on the quality of user inputs. The templates facilitate data analysis and support the scientific community towards going FAIR.

Standardizing FEM data collection is, besides a scientific necessity, a business need, aiming at improving the use of data within insurers. By having more robust datasets for evaluating occupational exposure hazards, insurers are better positioned to provide guidance on how to control these hazards, thereby reducing adverse health effects, leading to fewer claims [42].

\subsection{Responsible Persons and Data Use}

Reliability, quality and applicability of data generation and capturing strongly depends on the communication among the data shepherd, the data creators and the data analysts [26]. Comprehensive exposure measurements are often challenging to reproduce because contextual information and metadata descriptions are missing. Communication 
and uncertainty analyses are often neglected as not as important as conducting laboratory experiments per se. The main responsibilities:

- For the data shepherd are facilitating the communication and understanding between the stakeholders of the process parameters, materials, working practices and the data to be captured.

- For the data creator are measurement of concentrations in relevant locations, evaluating the goodness of the concentration measurements, and reporting of the required metadata and underlying assumptions, such as identified sources and mixing of concentrations.

- For the data analysts are to link contextual information and concentration measurements, showing how the pre-process, process, and post-process levels are associated with the work activity to present assumptions in mass flow analysis, to evaluate links of regional concentrations and personal exposure and to report/justify uncertainty.

The metadata description and annotation part increase the interoperability and reusability as per the FAIR principles, as they explain the context under which the data were captured [43]. At the same time, the inclusion of sufficient metadata allows for potential re-users to decide whether they can use the data under contexts different to those originally intended [26]. We combined and integrated different ontological IDs from diverse libraries (such as Exposure Ontology (https://bioportal.bioontology.org/ontologies/EXO (accessed on 5 June 2021)), eNanoMapper (https: / / bioportal.bioontology.org/ontologies / ENM (accessed on 5 June 2021)), Ontology of Consumer Health Vocabulary (https:// bioportal.bioontology.org/ontologies/OCHV (accessed on 5 June 2021)), NanoParticle Ontology (https:/ / bioportal.bioontology.org/ontologies/NPO (accessed on 5 June 2021)), PLOS Thesaurus (https://bioportal.bioontology.org/ontologies/PLOSTHES (accessed on 5 June 2021), etc.) when a single annotation from a source was missing [44]. A harmonized annotation including all descriptors is needed so that users can accurately identify and describe the processes causing emissions. Armiento et al. [39] demonstrated a two-step approach for ontologies extension using NanoParticle and eNanoMapper ontologies that lead to the addition of new concepts. The authors revealed that one issue that arises around ontologies is the degree of granularity usage during interpretation. A single schema focusing on reporting data elements is missing and it necessitates community consensus and the formulations of detailed recommendations on the metadata reporting requirements and well-defined words.

\subsection{Comparison with GRACIOUS Data Collection Sheets}

Recently, the GRACIOUS project (EU H2020, EC-GA No.760840) released templates for release, fate and exposure data collection [15]. Despite sharing a number of concepts and determinants, some key differences should be noted:

- We dedicate a tab for the user to insert protocol information used during the sampling such as for TEM or SEM offline analysis, or the sampling of gravimetric filters. An increased availability of validated protocols is necessary to advance both computational studies and material characterization through homogeneous data $[45,46]$. Sample preparation (such as duration of sample preparation, and solvents use) is an important step of any chemical analysis. The data creator is responsible to report relevant steps in the sample collection and analysis.

- Regarding the challenges of FAIRification, research institutions are often not sufficiently equipped with the specific knowhow and a number of researchers perceive these process requirements as additional paperwork that does not directly benefit their work [27]. Thus, the presented data collection sheet puts emphasis on the most essential elements to be captured, bypassing intense data reporting.

- The GRACIOUS template requires detailed p-chem characterization-for example, the moisture of NOs or aspect ratio. Moisture content alone cannot be used to predict environmental effects, i.e., the powder dustiness, because storing conditions also affect the dustiness [47]. NOs' length quantification is currently not feasible at sufficient 
accuracy from workplace air samples; only indicative dimensions and concentrations can be provided $[7,48]$. Thus, we focus our $\mathrm{p}$-chem characterization data on emission determination of offline analysis and not as an integral part of material characterization per se [4]. The difference refers to timing; the GRACIOUS template focuses on the p-chem of NOs before they are incorporated into the matrix, while we are interested in the characterization of released airborne NOs from offline analysis. Measurements and analyses not only need to be standardized, but also need to be made simpler, feasible and to some extent, cost efficient [12].

- A detail metadata description is provided to report the sampling strategy, measurement data and characterization part in an annotated manner to increase interoperability.

- Some variables not found in the GRACIOUS template include random air speed, local control efficiency and production rate (work intensity). Random air speed related to the process should be considered when estimating the dispersion of particles between NF and FF. LEV integrated in the process machine should be evaluated separately (e.g., when air is returning to room or exhausted outdoors, etc.). In addition, we invite and encourage data creators to add pictures and visualization files to help the next users, such as schemes of process and location of instrumentations. This helps data users to apply the data accordingly for their purpose.

- A FEM template should be flexible enough to include not prescribed factors that are process-specific, such as speed, temperature, pressure, the number of nozzles during a spray coating process or the ink viscosity in screen printing processes. This should allow the user to adjust the template in a process-specific manner.

- Concentration and emission data should be kept distinguishable. Emission depends on process parameters, while concentration depends both on emissions and environmental descriptors. We divide the process information-related data with the measurement requirements in clear and simple ways. We also limit the template to the FEM data without projecting assumptions to capture routes of exposure or potential of inhalation and dermal exposure. We provide a bottom-up designed template close to the raw data of exposure and emission assessments.

- NO fragments' properties depend on the release process described in the release experiment. We do not capture release experimentations. Products have intentional use applications, and the emissions are based on those. For example, Ag-NPs applied to textiles for clothes and toys have leaching to washing liquid, sweat and saliva as relevant release pathways but textiles for outdoor use have weathering (UV, abrasion, leaching) as a relevant release pathway. Thus, a template dedicated to release emissions and characterization of released fragments is more suitable.

- GRACIOUS templates extend to regulatory requirements of chemical risk assessment. ECHA descriptors are designed mainly for traditional industry where the process categories (PROCS) can be difficult to associate with nanotechnology industry processes. Those features were not included in our template. The main ECHA descriptors are article category (AC), exposure scenarios (ESs), and PROCs. These are combined to contributing activities (CA) that are used to describe release from an $A C$ in specific ESs by different PROCs. The CA, ES, and PROC descriptors with the quantitative NO release can be used for a "Lifecycle release and exposure" grouping strategy and parameterization of exposure and release models. AC defines where the substance has been processed (e.g., wood articles, plastic articles) and we maintained this information as the most relevant to the field campaign purpose. However, the categories are not well specified considering release. For example, ECHA AC1 is "Vehicles", which is difficult to estimate where or for which vehicle the substance has been processed. Better sub-categories are needed which can be further grouped based on, e.g., matrix properties that behave in similar way under certain release circumstances (e.g., AC13 plastic articles: AC13.1 poly(methyl methacrylate), AC13.2 polypropylene, AC13.3 polyamide). 


\subsection{Comparison with the eNanoMapper and NECID Database}

The eNanoMapper database provides a FAIR-aligned Nanosafety Data Interface ( https:/ / search.data.enanomapper.net/ (accessed on 5 June 2021)), with an aggregated search application to find data across diverse database instances [49]. The NanoReg2 database, included in eNanoMapper, is freely available and includes twelve datasets comprising of occupational exposure data derived from the Nano Exposure and Contextual Information Database (NECID) (https: / / perosh.eu/repository/necid-demo-version/ (accessed on 5 June 2021)) database. The data reporting format is not homogenized, and the structure reasoning is not reported. Data are missing and metadata description is not provided (see Supplementary Materials, Nanoreg2). For the moment, the exposure data are not yet in a reusable format; however, tremendous efforts have been put into making data re-usable. NECID focuses on describing operational conditions and working practices at a highly contextual information level. It covers an extensive number of parameters and descriptors that are specific to the workplace, worker, and measurements. NECID accepts raw measurement data with information about the instruments and sampling methods. In ASINA, we consider that it is the data analyst's responsibility to analyze the exposure determinants together with the data creator and partners. This ensures better communication and increases the data's reliability. This also significantly simplifies data collection requirements and ensures direct applicability of the data by users.

The NECID database is missing some of the most relevant contextual information considering worker exposure, such as material process/handling rate, production rates, emission control efficiency and random air flow at workstations. For example, one of the most relevant exposure determinants is the random air flow at the workstation diluting the concentrations after LEV [10]. For LEV, it is relevant to know if replacement air is extracted from the workstation or supplied elsewhere. This has a significant effect on the workstation concentration levels, regardless of the LEV capturing efficiency. Without such information, the results are not easy to be used to extrapolate other situations with sufficient precision. NECID rely more on qualitative exposure taxonomy than quantitative. Qualitative descriptors should be avoided in data collection because they are subjected to the interpretation of the data creator and analyst and the user, unless clearly defined and standardized. Categorization is useful when data are not available or the relevance considering the exposure or exposure extrapolation is not significant. However, in categorization, quantitative ranges should be provided to avoid misinterpretation of the categories.

Jeliazkova et al. [49] provide a detailed description of the eNanoMapper interface, the data included within and the path towards FAIRifying data (challenges and recommendations). The authors stress the lack of structured data available for re-use purposes and the need for user-friendly reporting formats, complete metadata description, ontology IDs and a harmonized terminology. The authors also recognize that available data are poor for computational purposes. It should be noted that exposure data in eNanoMapper represent the minority of safety data (40 out of 123.695 studies in total), showing that there is still a long road to make the safety aspect of occupational environment visible in the collective picture of nanosafety. The terminology, metadata description and ontology used in this template could accelerate the community's pace on the FAIR path.

\subsection{The Data Shepherd}

From a data shepherd perspective, the most challenging part was to leap from the outlined information of the questionnaire to the comprehensive state of a filled-in readyto-use data template. Data creators, for example, did not mention the importance of differentiating the location of measurements and the challenges in combining exposure and emission assessment. Gaining understanding in different but adjacent fields demands multiple collaborations from diverse partners for the shepherd [27]. Data shepherding as a novel concept and role in the field of nanotechnology is just forming [26]. Confusion may appear that the shepherd is a mediator for a partner to request more measurements from experimentalists, which is not the case. Communicating the needs and requirements of each 
stakeholder in a simple and understandable way is the core of the role and distances had to be kept from data management and data generation requirements. The data shepherd does not interfere with the experimental design plan but facilitates the information flow and ensures proper data and metadata capturing.

Burdened with the overview of all the project data, general comprehension of the relevant fields, identification of data creators, analysts, spreading the awareness of the value of FAIR data, ontology search, efficient data description communication management, actual template consensus realization and FAIRification of the data, the shepherd is a fulltime dedicated role. Successful data shepherding of a multi-partner scientific research project requires distinct functioning and resources.

\section{Conclusions}

The data and metadata curation topic, including its central role to nanoinformatics, workflow, data completeness and quality, has been the focus of multiple collaborative efforts and publications in the nano-field. Data shepherds have been introduced as a new role in the process of data FAIRification. We revealed the role challenges such as the multiple inner communications with a variety of stakeholders and the requirement of data overview understanding across several disciplines. We conclude that confusion of the new role as someone who defines the data requirements is perceived. It is essential to stress that the data shepherd does not interfere with the experimental design plan, and instead, streamlines information flow and ensures appropriate data and metadata capturing. All parties work together towards minimum, essential data requirements.

The paper enriches the scientific paradigm with valuable practical knowledge. We demonstrated the execution of a proposed framework, describing the steps taken in the development of a template for managing field monitoring data. The template and guidance are beneficial primarily for data creators, for reporting findings in a FAIR way and for data managers and analysts, for comprehending the actual processes behind the data and reducing data uncertainty. The ad hoc collaboration between stakeholders and the shepherd allowed the generation of a consensus template. The role of the shepherd without the support of all parties cannot be realized and, in this paper, we steer the role to evolve in the right direction.

We stress the fact that field monitoring data have rarely been a subject for re-use. This template can be enriched with studies from the literature and present the knowledge that exists in a systematic way. Future data creators and shepherds can capture data in a FAIR way. The templates must be dynamic, subject to differentiation depending on the goals of a project. Furthermore, accommodating extensive metadata in the template realizes the opportunity for collected data to serve additional purposes. Multi-exploitation is a significant scope of managing data FAIRly. The road towards creating effective FAIR templates can be readily adopted in other fields dealing with potentially hazardous materials.

Supplementary Materials: The following are available online at https:/ /www.mdpi.com/article/10 .3390/nano11071818/s1, S1: Template, S2: Nanoreg2 data.

Author Contributions: Conceptualization, I.F. and A.J.K.; methodology, I.F., A.A., F.M. and A.J.K.; validation, S.T. and B.D.S.; investigation, I.F. and A.J.K.; resources, S.T. and B.D.S.; data curation, I.F., S.T. and B.D.S.; writing — original draft preparation, I.F.; writing—review and editing, I.F., A.A., F.M. and A.J.K.; visualization, I.F.; supervision, F.M.; All authors have read and agreed to the published version of the manuscript.

Funding: This research was funded by the European Union's Horizon 2020 research and innovation pro-gramme under grant agreement No 862444.

Data Availability Statement: The raw data files are being uploaded into the NanoCommons Knowledge Base (https:/ / ssl.biomax.de/nanocommons/cgi/login_bioxm_portal.cgi (accessed on 5 June 2021)). 
Acknowledgments: The authors would like to thank Franco Belosi, Magda Blosi, and Fabrizio Ravegnani from the Institute of Atmospheric Sciences and Climate (ISAC) of the National Research Council (CNR) for their valuable knowledge exchange on monitoring data. The authors would also like to thank Anastasios G. Papadiamantis of NanoCommons for helping with data annotation and the Transnational Access Activities in general of the Horizon 2020 e-infrastructure project NanoCommons (Grant agreement no.: 731032).

Conflicts of Interest: The authors declare no conflict of interest.

\section{References}

1. Zhu, H.; Jiang, S.; Chen, H.; Roco, M.C. International perspective on nanotechnology papers, patents, and NSF awards (2000-2016). J. Nanopart. Res. 2017, 19, 370. [CrossRef]

2. Singh, N.A. Nanotechnology innovations, industrial applications and patents. Environ. Chem. Lett. 2017, 15, 185-191. [CrossRef]

3. Vaquero, C.; Gutierrez-Cañas, C.; Galarza, N.; De Ipina, J.L. Exposure assessment to engineered nanoparticles handled in industrial workplaces: The case of alloying nano- $\mathrm{TiO}_{2}$ in new steel formulations. J. Aerosol Sci. 2016, 102, 1-15. [CrossRef]

4. Kuhlbusch, T.A.J.; Wijnhoven, S.W.P.; Haase, A. Nanomaterial exposures for worker, consumer and the general public. NanoImpact 2018, 10, 11-25. [CrossRef]

5. Huguet-Casquero, A.; Gainza, E.; Pedraz, J.L. Towards Green Nanoscience: From extraction to nanoformulation. Biotechnol. Adv. 2021, 46, 107657. [CrossRef]

6. Saleem, H.; Zaidi, S.J. Sustainable Use of Nanomaterials in Textiles and Their Environmental Impact. Materials 2020, $13,5134$. [CrossRef]

7. Koivisto, A.J.; Bluhme, A.B.; Kling, K.I.; Fonseca, A.S.; Redant, E.; Andrade, F.; Krepker, M.; Segal, E.; Vogel, U.; Koponen, I.K.; et al. Occupational exposure during handling and loading of halloysite nanotubes-A case study of counting nanofibers. NanoImpact 2018, 10, 153-160. [CrossRef]

8. Viitanen, A.-K.; Uuksulainen, S.; Koivisto, A.J.; Hämeri, K.; Kauppinen, T. Workplace Measurements of Ultrafine Particles-A Literature Review. Ann. Work Expo. Health 2017, 61, 749-758. [CrossRef]

9. OECD. Harmonized tiered approach to measure and assess the potential exposure to airborne emissions of engineered nanoobjects and their agglomerates and aggregates at workplaces. In Series on the Safety of Manufactured Nanomaterials; OECD: Paris, France, 2015.

10. Koivisto, A.; Spinazzè, A.; Verdonck, F.; Borghi, F.; Löndahl, J.; Koponen, I.K.; Jayjock, M.; Hussein, T.; Arnold, S.; Furxhi, I.; et al. Assessment of exposure determinants and exposure levels by using stationary concentration measurements and a probabilistic near-field/far-field exposure model [version 1; peer review: Awaiting peer review]. Open Res. Eur. 2021. [CrossRef]

11. European Union. Revision of EU Legislation on Registration, Evaluation, Authorisation and Restriction of Chemicals; European Union: Brussels, Belgium, 2021; p. 4.

12. Koivisto, A.J.; Kling, K.I.; Hänninen, O.; Jayjock, M.; Löndahl, J.; Wierzbicka, A.; Uhrbrand, K.; Viana, M.; Morawska, L.; Hussein, T.; et al. Source specific exposure and risk assessment for indoor aerosols. Sci. Total Environ. 2019, 668, 13-24. [CrossRef]

13. Boccuni, F.; Ferrante, R.; Tombolini, F.; Lega, D.; Antonini, A.; Alvino, A.; Pingue, P.; Beltram, F.; Sorba, L.; Iavicoli, S.; et al. Workers' Exposure to Nano-Objects with Different Dimensionalities in R\&D Laboratories: Measurement Strategy and Field Studies. Int. J. Mol. Sci. 2018, 19, 349.

14. Fonseca, A.S.; Viitanen, A.K.; Kanerva, T.; Säämänen, A.; Aguerre-Chariol, O.; Fable, S.; Dermigny, A.; Karoski, N.; Fraboulet, I.; Koivisto, J.; et al. Occupational Exposure and Environmental Release: The Case Study of Pouring $\mathrm{TiO}_{2}$ and Filler $\mathrm{Materials}$ for Paint Production. Int. J. Environ. Res. Public Health 2021, 18, 418. [CrossRef] [PubMed]

15. Shahzad, R.; Veronique, A.; Ana, S.F.; Socorro, V.; Camila, D.; Araceli, S.J.; Nina, J. GRACIOUS Release and Exposure Templates. Available online: https:/ / zenodo.org/record/4665253\#.YOlza-hKguU (accessed on 5 June 2021).

16. van Vlijmen, H.; Mons, A.; Waalkens, A.; Franke, W.; Baak, A.; Ruiter, G.; Kirkpatrick, C.; da Silva Santos, L.O.B.; Meerman, B.; Jellema, R.; et al. The Need of Industry to Go FAIR. Data Intell. 2020, 2, 276-284. [CrossRef]

17. Wise, J.; de Barron, A.G.; Splendiani, A.; Balali-Mood, B.; Vasant, D.; Little, E.; Mellino, G.; Harrow, I.; Smith, I.; Taubert, J.; et al. Implementation and relevance of FAIR data principles in biopharmaceutical R\&D. Drug Discov. Today 2019, $24,933-938$.

18. European Union. Chemicals Strategy for Sustainability Towards a Toxic-Free Environment. In Communication from the Com-mission to the European Parliament, the Council, the European Economic and Social Committee and the Committee of the Regions; European Union: Brussels, Belgium, 2020.

19. European Commission. Communication from the Commission to the European Parliament, the European Council, the Council, the European Economic and Social Committee and the Committee of the Regions a New Industrial Strategy for Europe; European Commission: Brussels, Belgium, 2020.

20. Boeckhout, M.; Zielhuis, G.A.; Bredenoord, A.L. The FAIR guiding principles for data stewardship: Fair enough? Eur. J. Hum. Genet. 2018, 26, 931-936. [CrossRef]

21. European Commission. H2020 Programme: Guidelines on FAIR Data Management in Horizon 2020; Directorate-General for Re-search \& Innovation. 2016. Available online: https:/ / ec.europa.eu/research/participants/data/ref/h2020/grants_manual/ hi/oa_pilot/h2020-hi-oa-data-mgt_en.pdf (accessed on 13 July 2021). 
22. Romanos, N.; Kalogerini, M.; Koumoulos, E.P.; Morozinis, A.K.; Sebastiani, M.; Charitidis, C. Innovative Data Management in advanced characterization: Implications for materials design. Mater. Today Commun. 2019, 20, 100541. [CrossRef]

23. Wilkinson, M.D.; Dumontier, M.; Aalbersberg, I.J.; Appleton, G.; Axton, M.; Baak, A.; Zhao, J.; Wittenburg, P.; Waagmeester, A.; Mons, B.; et al. The FAIR Guiding Principles for scientific data management and stewardship. Sci. Data 2016, 3, 160018. [CrossRef]

24. Jacobsen, A.; de Miranda Azevedo, R.; Juty, N.; Batista, D.; Coles, S.; Cornet, R.; Strawn, G.; Thompson, M.; Roos, M.; Schultes, E.; et al. FAIR Principles: Interpretations and Implementation Considerations. Data Intell. 2020, 2, 10-29. [CrossRef]

25. Bloemers, M.; Montesanti, A. The FAIR Funding Model: Providing a Framework for Research Funders to Drive the Transition toward FAIR Data Management and Stewardship Practices. Data Intell. 2020, 2, 171-180. [CrossRef]

26. Papadiamantis, A.G.; Klaessig, F.C.; Exner, T.E.; Hofer, S.; Hofstaetter, N.; Himly, M.; Doganis, P.; Afantitis, A.; Melagraki, G.; Lynch, I.; et al. Metadata Stewardship in Nanosafety Research: Community-Driven Organisation of Metadata Schemas to Support FAIR Nanoscience Data. Nanomaterials 2020, 10, 2033. [CrossRef]

27. Furxhi, I.; Arvanitis, A.; Murphy, F.; Costa, A.; Blosi, M. Data Shepherding in Nanotechnology. The Initiation. Nanomaterials 2021, 11, 1520. [CrossRef]

28. Powers, C.M.; Mills, K.A.; Morris, S.A.; Klaessig, F.; Gaheen, S.; Lewinski, N.; Hendren, C.O. Nanocuration workflows: Establishing best practices for identifying, inputting, and sharing data to inform decisions on nanomaterials. Beilstein J. Nanotechnol. 2015, 6, 1860-1871. [CrossRef]

29. Koivisto, J. Source Specific Risk Assessment of Indoor Aerosol Particles. Ph.D. Thesis, University of Helsinki (Helsingin yliopisto), Helsinki, Finland, 2013.

30. Koivisto, A.J.; Kling, K.I.; Fonseca, A.S.; Bluhme, A.B.; Moreman, M.; Yu, M.; Costa, A.L.; Giovanni, B.; Ortelli, S.; Jensen, K.A.; et al. Dip coating of air purifier ceramic honeycombs with photocatalytic TiO2 nanoparticles: A case study for occupational exposure. Sci. Total Environ. 2018, 630, 1283-1291. [CrossRef] [PubMed]

31. European Chemicals Agency. Guidance on Information Requirements and Chemical Safety Assessment Chapter R.14: Occupational Exposure Assessment; European Chemicals Agency: Helsinki, Finland. 2016. Available online: https://echa. europa.eu/documents/10162/13632/information_requirements_r14_en.pdf/bb14b581-f7ef-4587-a171-17bf4b332378 (accessed on 13 July 2021).

32. Koivisto, A.J.; Jensen, A.C.Ø.; Kling, K.I.; Nørgaard, A.; Brinch, A.; Christensen, F.; Jensen, K.A. Quantitative material releases from products and articles containing manufactured nanomaterials: Towards a release library. NanoImpact 2017, 5, 119-132. [CrossRef]

33. Fransman, W.; Schinkel, J.; Meijster, T.; Van Hemmen, J.; Tielemans, E.; Goede, H. Development and evaluation of an exposure control efficacy library (ECEL). Ann. Occup. Hyg. 2008, 52, 567-575. [PubMed]

34. Goede, H.; Christopher-de Vries, Y.; Kuijpers, E.; Fransman, W. A Review of Workplace Risk Management Measures for Nanomaterials to Mitigate Inhalation and Dermal Exposure. Ann. Work Exp. Health 2018, 62, 907-922. [CrossRef]

35. Baldwin, P.E.; Maynard, A.D. A Survey of Wind Speeds in Indoor Workplaces. Ann. Occup. Hyg. 1998, 42, 303-313. [CrossRef]

36. Organisation for Economic Co-Operation and Development. Strategies, techniques and sampling protocols for determining the concentrations of manufactured nanomaterials in air at the workplace. In Series on the Safety of Manufactured Nanomaterials; Organisation for Economic Co-Operation and Development: Paris, France, 2017; p. 155.

37. Harper, M. Recent Advances in Occupational Exposure Assessment of Aerosols. Int. J. Environ. Res. Public Health 2020, 17, 6820. [CrossRef]

38. Levin, M.; Witschger, O.; Bau, S.; Jankowska, E.; Koponen, I.K.; Koivisto, A.J.; Clausen, P.A.; Jensen, A.; Mølhave, K.; Asbach, C.; et al. Can We Trust Real Time Measurements of Lung Deposited Surface Area Concentrations in Dust from Powder Nanomaterials? Aerosol Air Qual. Res. 2016, 16, 1105-1117. [CrossRef]

39. Huanyu, L.; Armiento, R.; Lambrix, P. Method for Extending Ontologies with Application to the Materials Science Domain. Data Sci. J. 2019, 18, 50 .

40. Kochev, N.; Jeliazkova, N.; Paskaleva, V.; Tancheva, G.; Iliev, L.; Ritchie, P.; Jeliazkov, V. Your Spreadsheets Can Be FAIR: A Tool and FAIRification Workflow for the eNanoMapper Database. Nanomaterials 2020, 10, 1908. [CrossRef]

41. Clark, K.; van Tongeren, M.; Christensen, F.M.; Brouwer, D.; Nowack, B.; Gottschalk, F.; Micheletti, C.; Schmid, K.; Gerritsen, R.; Aitken, R.; et al. Limitations and information needs for engineered nanomaterial-specific exposure estimation and scenarios: Recommendations for improved reporting practices. J. Nanopart. Res. 2012, 14, 970. [CrossRef]

42. Shockey, T.M.; Babik, K.R.; Wurzelbacher, S.J.; Moore, L.L.; Bisesi, M.S. Occupational exposure monitoring data collection, storage, and use among state-based and private workers' compensation insurers. J. Occup. Environ. Hyg. 2018, 15, 502-509. [CrossRef] [PubMed]

43. Karcher, S.; Willighagen, E.L.; Rumble, J.; Ehrhart, F.; Evelo, C.T.; Fritts, M.; Gaheen, S.; Harper, S.L.; Hoover, M.D.; Jeliazkova, N.; et al. Integration among databases and data sets to support productive nanotechnology: Challenges and recommendations. NanoImpact 2018, 9, 85-101. [CrossRef]

44. Samuel, J. Collaborative Approach to Developing a Multilingual Ontology: A Case Study of Wikidata; Springer International Publishing: Cham, Switzerland, 2017.

45. Johnston, L.J.; Gonzalez-Rojano, N.; Wilkinson, K.J.; Xing, B. Key challenges for evaluation of the safety of engineered nanomaterials. NanoImpact 2020, 18, 100219. [CrossRef] 
46. Furxhi, I.; Murphy, F. Predicting In Vitro Neurotoxicity Induced by Nanoparticles Using Machine Learning. Int. J. Mol. Sci. 2020, 21, 5280. [CrossRef] [PubMed]

47. Levin, M.; Rojas, E.; Vanhala, E.; Vippola, M.; Liguori, B.; Kling, K.I.; Koponen, I.K.; Mølhave, K.; Tuomi, T.; Gregurec, D.; et al. Influence of relative humidity and physical load during storage on dustiness of inorganic nanomaterials: Implications for testing and risk assessment. J. Nanopart. Res. 2015, 17, 337. [CrossRef]

48. Fonseca, A.S.; Viitanen, A.K.; Koivisto, A.J.; Kangas, A.; Huhtiniemi, M.; Hussein, T.; Vanhala, E.; Viana, M.; Querol, X.; Hämeri, K.; et al. Characterization of exposure to carbon nanotubes in an industrial setting. Ann. Occup. Hyg. 2015, 59, 586-599. [PubMed]

49. Jeliazkova, N.; Apostolova, M.D.; Andreoli, C.; Barone, F.; Barrick, A.; Battistelli, C.; Bossa, C.; Botea-Petcu, A.; Châtel, A.; De Angelis, I.; et al. Towards FAIR nanosafety data. Nat. Nanotechnol. 2021, 16, 644-654. [CrossRef] 